\title{
Coding and long noncoding transcriptomic responses of Emiliania huxleyi to viral infection
}

\author{
ENQUAN ZHANG, WEICONG CAI, AND JINGWEN LIU*
}

College of Food and Bioengineering, Jimei University, Xiamen 361021, China (* correspondence: 1jwsbch@163.com)

\section{Methods and results}

We analyzed the global transcriptome changes of marine phytoplankton Emiliania huxleyi in response to its specific virus (EhV). As a result, a total of 14695 mRNAs and 4294 lncRNAs were differentially expressed at $6 \mathrm{~h}$ and $45 \mathrm{~h}$ post-infection. KEGG enrichment analysis of DE lncRNAs showed that some metabolic pathaways were regulated by lncRNAs, including fatty acid metabolism, steroid metabolism, terpenoid backbone biosynthesis and so on. These pathways were shown in the figure below.

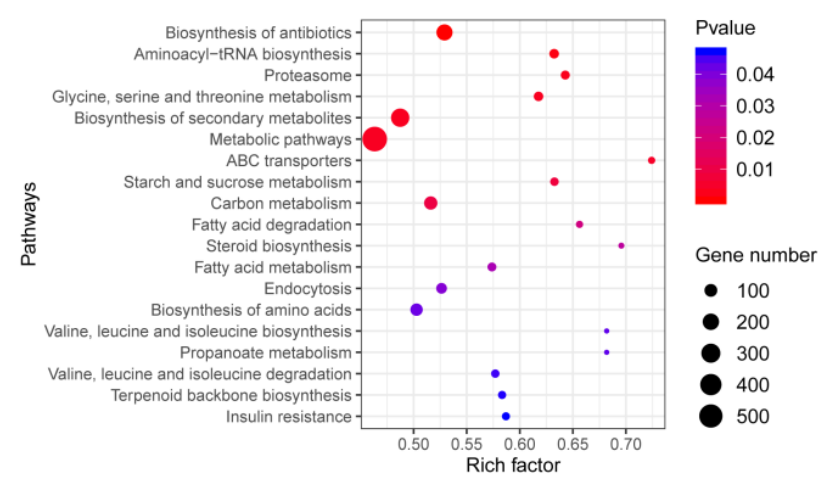

Figure 1: Pathway enrichment analysis of DE lncRNAs.

\section{Discussion of results}

Our transcriptomic results showed that there was a deep remodeling of transcripts associated with lipid metabolism under the condition of viral infection, indicating that lipids might be the key materials in the process of viral infection. This result was consistent with previous studies [1,2]. We identify a multitude of novel lncRNAs specifically responsive to viral infection, suggesting putative regulatory roles in the regulation of EhV infection. Twenty of these IncRNAs were validated by reverse transcription qPCR. Moreover, part of the DE mRNAs linked with lipid metabolism were predicted to be the target mRNAs of IncRNAs (correlation coefficient cutoff was 0.95), so we speculated that these mRNAs might be regulated by lncRNAs.

[1] Zeng et al. (2019) Sci Total Environ 688, 521-528. [2] Rosenwasser et al. (2014) Plant Cell 26, 2689-2707. 